\title{
The presence and sequence of endotracheal tube colonization in patients undergoing mechanical ventilation
}

\author{
C. Feldman*, M. Kassel**, J. Cantrell ${ }^{+}$, S. Kaka**, R. Morar*, A. Goolam Mahomed*, J.I. Philips ${ }^{+}$
}

The presence and sequence of endotracheal tube colonization in patients undergoing mechanical ventilation. C. Feldman, M. Kassel, J. Cantrell, S. Kaka, R. Morar, A. Goolam Mahomed, J.I. Philips. (C) ERS Journals Ltd 1999.

ABSTRACT: Endotracheal tube colonization in patients undergoing mechanical ventilation was investigated.

In the first part of this prospective study, the airway access tube was examined for the presence of secretions, airway obstruction and bacterial colonization, in cases undergoing extubation or tube change. In the second part of the study, the sequence of oropharyngeal, gastric, respiratory tract and endotracheal tube colonization was investigated by sequential swabbing at each site twice daily for 5 days in consecutive noninfected patients.

In the first part, it was noted that all airway access tubes of cases undergoing extubation had secretions lining the interior of the distal third of the tube which were shown on scanning electron microscopy to be a biofilm. Gram-negative micro-organisms were isolated from these secretions in all but three cases. In the second part, it was noted that the sequence of colonization in patients undergoing mechanical ventilation was the oropharynx $(36 \mathrm{~h})$, the stomach $(36-60 \mathrm{~h})$, the lower respiratory tract $(60-84 \mathrm{~h})$, and thereafter the endotracheal tube $(60-96 \mathrm{~h})$. Nosocomial pneumonia occurred in 13 patients and in eight cases identical organisms were noted in lower respiratory tract secretions and in secretions lining the interior of the endotracheal tube.

The endotracheal tube of patients undergoing mechanical ventilation becomes colonized rapidly with micro-organisms commonly associated with nosocomial pneumonia, and which may represent a persistent source of organisms causing such infections. Eur Respir J 1999; 13: 546-551.
*Intensive Care Unit, Dept of Medicine, University of the Witwatersrand, South Africa. **Dept of Medical Microbiology, South African Institute for Medical Research, University of the Witwatersrand, South Africa. 'National Centre for Occupational Health, Dept of Health, South Africa.

Correspondence: C. Feldman

Dept of Medicine

University of the Witwatersrand

7 York Road

Parktown, 2193

Johannesburg

South Africa

Fax: 27116438777

Keywords: Biofilm

colonization

endotracheal tube

Gram-negative bacteria oropharynx

respiratory secretions

Received: July 291998

Accepted after revision October 131998
In 1972, JoHANSON et al. [1] were the first to note an increase in oropharyngeal colonization by micro-organisms in patients with increasing severity of illness, and to document thereafter that there was an increased risk of the development of nosocomial pneumonia in patients who had Gram-negative oropharyngeal colonization. Subsequent to the findings by these investigators and many others, it has long been recognized that there is a direct relationship between oropharyngeal colonization and nosocomial pneumonia [2-9]. However, despite numerous studies there is still considerable ongoing debate as to the primary site of colonization, as well as the exact sources and routes of this colonization process [10-13]. Increasing research interest over the past few years has focused on the possible role of direct tracheal colonization [5, 7], as well as the potential role of the endotracheal tube (ETT) itself in the colonization process [14-17].

Bacterial adherence to objects, such as intravenous catheters, by means of the so-called biofilm, in the causation of various nosocomial infections, has been studied over several years [14, 15]. In 1986, SotTILE et al. [14] used scanning electron microscopy (SEM) and qualitative cultures to assess bacterial adhesion to the interior of polyvinylchloride ETTs. They examined biofilm colonization of ETTs, which they considered may act as a reservoir for contamination of the tracheobronchial tree [14]. In their study of 25 patients in the intensive care unit (ICU) under- going extubation, the ETT was completely covered in $84 \%$ of cases and partially covered in $16 \%$ by an amorphous bacterium-containing matrix [14]. They suggested that there may be a relationship between nosocomial pulmonary infections and bacterial adherence to the interior surface of the ETT [14]. Several investigators have subsequently studied this process further [15-17] and have suggested not only that the formation of a biofilm may be an important mechanism of ETT obstruction, but also a potential source of pulmonary infection $[16,17]$.

The main purpose of the current investigation was to determine more precisely the pattern and sequence of ETT colonization in patients in the ICU undergoing mechanical ventilation. In the first part of the study, the presence of colonization and biofilm formation within the interior of the ETT was examined, and in the second part ETT colonization was investigated simultaneously with oropharyngeal, gastric and lower respiratory tract colonization, to determine by which routes micro-organisms may colonize the ETT.

\section{Materials and methods}

\section{Patient data}

This was a prospective study conducted in two parts at the Hillbrow Hospital Intensive Care Unit, Johannesburg, 
South Africa. Permission to undertake these investigations was obtained from the Committee for Research on Human Subjects of the University of the Witwatersrand. The following data were recorded in each case: age and sex of the patient, reasons for mechanical ventilation, type of humidification used (humidifier in the ventilator circuit or heat and moisture exchanger), duration of ventilation, means of respiratory access (oral or nasal ETT or tracheostomy), method of stress ulcer prophylaxis (histamine type 2-receptor blockers or barrier preparation, i.e. sucralfate), the occurrence or not of nosocomial pneumonia, together with data of suspected nosocomial pathogens, using criteria similar to those previously described $[10,11]$.

The diagnosis of nosocomial pneumonia was based on the presence after $\geq 48 \mathrm{~h}$ of ventilation of all the following criteria: 1) radiological evidence of new pulmonary infiltrates; 2) fever of $>38^{\circ} \mathrm{C}$ taken rectally; 3) leukocytosis of $>10 \times 10^{9}$ cells $\cdot \mathrm{L}^{-1}$; 4) purulent tracheobronchial secretions; and 5) no evidence of infection elsewhere. Organisms cultured in significant amounts $(2+(20-100$ colonies $)$ and $3+(>100$ colonies) growth) from good quality specimens of secretions obtained by sterile suctioning of the lower respiratory tract through the ETT or tracheostomy tube were considered to be likely causative pathogens, as previously described [10].

\section{Part 1 of the study}

In the first part of the study, the airway access tubes (ETT, nasotracheal or tracheostomy tubes) of 21 consecutive patients undergoing extubation for either tube change or following mechanical ventilation were studied. On extubation, the interior of the tube was immediately sampled using a sterile throat swab, and these specimens submitted for microbiological analysis. Thereafter, the tubes were inspected for evidence of secretions that coated and/or obstructed the interior of the lumen, the latter being assessed by attempting to pass a standard ETT suction catheter through the lumen of the tube. The degree of tube obstruction (mild, moderate or severe) and the site of these secretions (distal, middle or proximal third of the tube) were recorded.

In addition, SEM of the secretions lining the interior of the ETT of a patient following extubation was performed using standard techniques [18]. Briefly, the distal half of the tube was placed in $3 \%$ gluteraldehyde and stored at $4{ }^{\circ} \mathrm{C}$ for $48 \mathrm{~h}$. After fixation, the tube was rinsed in Millonigs buffer. Samples were taken $1.5 \mathrm{~cm}$ from the tip, divided longitudinally, and processed for SEM as follows. Primary fixation was in 3\% gluteraldehyde in Millonigs buffer $(\mathrm{pH}$ 7.4 ) for $48 \mathrm{~h}$. Post-fixation was in $1 \%$ osmium tetroxide in Millonigs buffer ( $\mathrm{pH}$ 7.4) for $1 \mathrm{~h}$. This was followed by dehydration in graded ethanol 50, 70, 90 and $100 \%$. Two samples were dried in a Hitachi critical point apparatus (Hitachi HCP/2, Koki Company Ltd., Tokyo, Japan) and a further two samples placed in hexamethyldisilazane (HMDS) for $5 \mathrm{~min}$. The samples were then gold coated with an SEM Coating Unit E5100 (Polaron Cool Sputter Coater, Watford, UK) and viewed with a JSM 840 SEM (Jeol JSM 840, Jeol Pty Ltd, Tokyo, Japan). The critical point drying and HMDS methods were performed simultaneously to compare the result of the two methods, and the HMDS technique gave better results.
The swabs taken from the interior of the tube were plated onto blood and McConkey agar which were incubated and read after 24 and $48 \mathrm{~h}$. Each morphological type of colony present was picked off and identified according to standard microbiological protocols. The growth of micro-organisms was quantitated as follows: 1) no growth; 2) 1-5 colonies as scanty growth; 3) 6-20 colonies as 1+ growth; 4) 20-100 colonies as 2+ growth; and 5) $>100$ colonies as $3+$ growth. The isolation of organisms with $1+$ growth was considered to represent significant colonization. Susceptibility of all isolated micro-organisms to 14 antibiotic agents was measured using the disc diffusion method. These tests of antibiotic susceptibility were undertaken for comparative purposes, as a measure of the likelihood that the colonizing organisms were the same as those considered responsible for the nosocomial infections.

\section{Part 2 of the study}

In the second part of the study, 10 consecutive noninfected patients admitted to the ICU for mechanical ventilation, of a duration of at least 5 days, were recruited. Sampling of the oropharynx (using a sterile throat swab), nasogastric tube (through suction catheter), interior of the airway tube (throat swab) and endotracheal secretions (sterile suction catheter) was undertaken immediately on admission and at 08:00 $\mathrm{h}$ and 16:00 $\mathrm{h}$ daily for 5 days. All specimens were immediately plated onto McConkey agar only (to suppress and exclude commensal upper respiratory tract flora) and incubated and read after 24 and $48 \mathrm{~h}$. Each morphological type of colony present was picked off and identified according to standard microbiological protocols. The growth of micro-organisms was quantitated and the susceptibility to 14 antibiotic agents was measured in a similar manner to that described in part 1 of the study.

\section{Results}

\section{Part 1 of the study}

In the first part of the study, 21 patients were recruited who were extubated on 24 occasions. The duration of ventilation varied between $12 \mathrm{~h}$ and 12 days. The clinical features of these patients, as well as the reasons for mechanical ventilation are shown in table 1 . There was no apparent relationship between the type of humidification or method of stress ulcer prophylaxis used and the presence or absence or nature of tube secretions.

Secretions lining the interior of the ETT or tracheostomy tubes were noted in all cases, which was the cause of obstruction in 11 cases, including two tracheostomy tubes. These secretions were noted from as early as $12 \mathrm{~h}$ (in one patient in whom significant growth of Gram-negative organisms $(3+$ growth) were cultured from the secretions), but were more pronounced from $48 \mathrm{~h}$. The site of secretions was invariably in the distal third of the tube beginning just above the distal open end (10 cases) and in one tube additionally in the middle third, but never in the proximal portion. These secretions caused severe tube obstruction in one case (unable to pass a suction catheter beyond the secretions). 
Table 1. - Clinical features and reasons for mechanical ventilation of 21 patients enrolled in the first part of the study undergoing mechanical ventilation in the intensive care unit

\begin{tabular}{lc}
\hline Characteristics & Number \\
\hline Mean age (range) yrs & $42.2(25-86)$ \\
Male/female & $11 / 10$ \\
Duration of ventilation days & $0.5-12$ \\
Reasons for ventilation & \\
Respiratory failure & 14 \\
$\quad$ Pneumonia & 4 \\
$\quad$ Trauma & 4 \\
$\quad$ Polyneuropathy & 2 \\
$\quad$ Asthma & 2 \\
$\quad$ Drug overdose & 1 \\
$\quad$ Surgical sepsis & 1 \\
Postoperative & 7 \\
$\quad$ Surgery & 6 \\
Trauma & 1 \\
Airway access tube & \\
Orotracheal & 5 \\
Nasotracheal & 11 \\
Tracheostomy & 4 \\
Humidification & \\
Humidifier in the circuit & 15 \\
Heat and moisture exchanger & 6 \\
Stress ulcer prophylaxis & \\
Sucralfate & 11 \\
Histamine-2-blocker & 7 \\
Nil & 3 \\
\hline
\end{tabular}

Figures 1a and $\mathrm{b}$ are SEMs of the ETT biofilm lining the interior lumen of the airway access tube of a patient following extubation. In addition to demonstrating dense amorphous material covering most of the surface of the tube, there are clumps of material projecting into the lumen, as well as micro-organisms appearing to adhere to this matrix. Micro-organisms were isolated from these secretions in all but three tubes (patients mechanically ventilated for 3, 9 and 12 days, respectively). The organisms isolated (sometimes multiple) are shown in table 2 .

Nosocomial pneumonia was diagnosed in 10 of these patients between 5-12 days following initiation of mechanical ventilation. The organisms isolated (sometimes multiple) in secretions obtained by suctioning of the lower respiratory tract of these cases and deemed to be the likely cause of the pneumonia were Pseudomonas aeruginosa (6 cases), Acinetobacter anitratus (5 cases) Klebsiella pneumoniae (3 cases), Proteus mirabilis (3 cases) and Enterobacter spp. (1 case). As can be seen from table 2, in six of these patients at least one of the micro-organisms deemed to have been responsible for the previous nosocomial pneumonia was still found colonizing the ETT on extubation, following apparently successful treatment of the pneumonia.

\section{Second part of the study}

In the second part of the study, 10 male patients were recruited. The clinical features are shown in table 3. All patients had previously been well with no evidence on admission of any infection. In only one patient were no organisms isolated from any of the sites sampled over the 5-day period. Nine of the 10 cases subsequently became
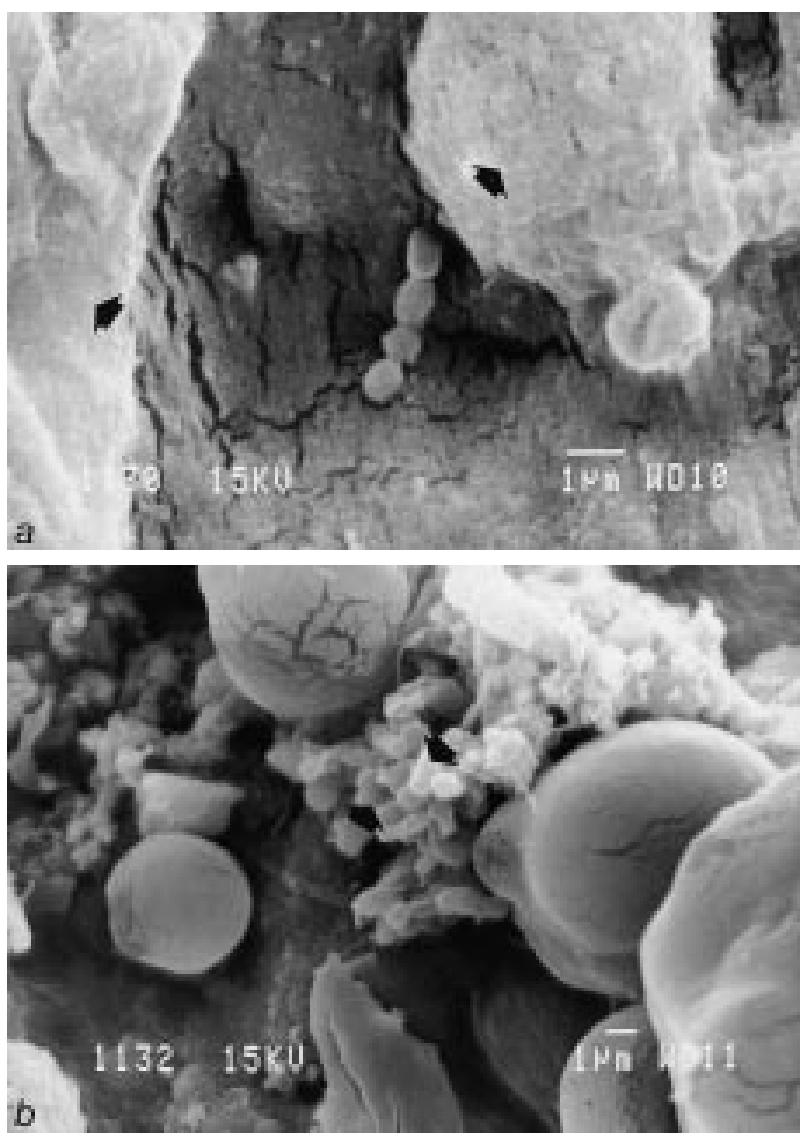

Fig. 1. - The appearance of a biofilm on scanning electron microscopy of the interior of an endotracheal tube of a patient following extubation. a) Shows the dense amorphous material covering much of the interior of the tube, with additional material projecting into the lumen of the tube (arrow). b) Shows micro-organisms apparently adhering to the amorphous matrix (arrow).

colonized with various micro-organisms, Gram-negative bacilli being the most common (A. anitratus, $K$. pneumoniae, $P$. aeruginosa, Escherichia coli and Enterobacter spp.), although Gram-positive micro-organisms were also sometimes noted (Staphylococcus aureus, S. epidermidis and Streptococcus bovis).

The isolation of colonizing organisms from the various sites sampled appeared to follow a set pattern, whether these were Gram-positive or Gram-negative isolates. The oropharynx was the first site to be colonized most commonly at $36 \mathrm{~h}$. Thereafter, the stomach became colonized $(36-60 \mathrm{~h})$ and then the lower respiratory tract (beginning at $36 \mathrm{~h}$, but being most abundant between 60-84 h). Isolation of organisms from the ETT itself began at $48 \mathrm{~h}$ (scanty isolation), but occurred in significant amounts from 60 $96 \mathrm{~h}$.

While the numbers of individual isolates were very small, there was a tendency for Gram-positive organisms to colonize the oropharynx early, between $12-36 \mathrm{~h}$, and to appear rapidly thereafter (12-24 $\mathrm{h}$ later, respectively) in the stomach and then in secretions from the lower respiratory tract. No Gram-positive isolates were found to colonize the ETT in significant amounts (only scanty growth). Colonization of the oropharynx with A. anitratus occurred later, between $36-48 \mathrm{~h}$, and these organisms progressively colonized the stomach, thereafter appearing in endotracheal 
Table 2. - Micro-organisms (sometimes multiple) isolated from the interior of the airway access tube of patients undergoing mechanical ventilation in the intensive care unit

Part 1: following extubation or tube change

Acinetobacter anitratus

Klebsiella pneumoniae

Pseudomonas aeruginosa

Proteus mirabilis

Escherichia coli

Enterobacter spp.

Morganella morgani

Enterococcus faecalis

Streptococcus viridans

Staphylococcus aureus

Part 2: during mechanical ventilation

A. anitratus

K. pneumoniae

Enterobacter spp.

secretions and all these isolates were subsequently noted within the ETT by $96 \mathrm{~h}$. The pattern of colonization with Pseudomonas spp. and Klebsiella spp. was not clear-cut. Other Enterobacteriaceae colonized the oropharynx and stomach from $48 \mathrm{~h}$, only occasionally appearing in the lower respiratory tract and in endotracheal secretions. Figure 2 shows the time sequence of colonization of the oropharynx, stomach, lower respiratory tract and ETT in four patients colonized with $A$. anitratus and four patients colonized with staphylococcal isolates.

Nosocomial pneumonia was diagnosed in three patients, occurring after $48 \mathrm{~h}$. In one case, $P$. aeruginosa was deemed to be the causative pathogen, being cultured from lower respiratory tract secretions. In this patient, no organisms were isolated from within the ETT. In two cases, $A$. anitratus was deemed responsible for the infection. In both patients, the organisms isolated from the lower respiratory tract were identical to those isolated from the interior of the ETT, as documented by same speciation and identical antimicrobial susceptibility patterns. In one of the cases significant colonization of the ETT and lower respiratory tract by $A$. anitratus occurred simultaneously at $60 \mathrm{~h}$, and was followed by clinical evidence of nosocomial pneumonia at $96 \mathrm{~h}$. In the second case, significant colonization of the lower respiratory tract by this organism occurred at $72 \mathrm{~h}$, and of the ETT at $84 \mathrm{~h}$, followed by clinical evidence of nosocomial pneumonia at $96 \mathrm{~h}$.

\section{Site of colonization}

\begin{tabular}{|c|c|c|c|c|c|c|c|c|}
\hline Oropharynx & $\therefore$ & :oo & $\because 000$ & $\bullet \bullet \bullet$ & & & & \\
\hline Gastric & & 。 & $\therefore$ & $\ddot{\circ}$ & @o:० & $0^{\bullet \bullet \bullet \bullet}$ & & \\
\hline LRT & & & $\circ$ & $\circ$ & $\because$ & $\because 0 \bullet$ & $\because \infty \circ$ & \\
\hline ETT & & & & & • & $\bullet$ & $\bullet$ & $\bullet \bullet \bullet \bullet$ \\
\hline
\end{tabular}

Fig. 2. - The time sequence of colonization of the oropharynx, the stomach (gastric), the lower respiratory tract (LRT) and the endotracheal tube (ETT) by Acinetobacter anitratus (Gram-negative isolates; $\bullet$ ) in four patients and by staphylococcal isolates (Gram-positive isolates; $\bigcirc)$ in four patients.
Table 3. - Clinical features and reasons for mechanical ventilation of 10 patients enrolled in the second part of the study undergoing mechanical ventilation in the intensive care unit

\begin{tabular}{lc}
\hline Characteristics & Number \\
\hline Mean age (range) yrs & $34.9(18-61)$ \\
Male/female & $10 / 0$ \\
Reasons for ventilation & 10 \\
$\quad$ Trauma & \\
$\begin{array}{l}\text { Airway access tube } \\
\quad \text { Orotracheal }\end{array}$ & 10 \\
Humidification & 10 \\
$\quad$ Heat and moisture exchanger & \\
Stress ulcer prophylaxis & 3 \\
$\quad$ Sucralfate & 7 \\
$\quad$ Histamine-2-receptor blocker & \\
\hline
\end{tabular}

\section{Discussion}

In this study, it was documented that the interior of the ETT of patients undergoing mechanical ventilation rapidly became colonized with Gram-negative micro-organisms which commonly appeared to survive within a biofilm. While it appears that colonization of the ETT may begin from as early as $12 \mathrm{~h}$, it is most abundant at $96 \mathrm{~h}$. This investigation further suggests that the common sequence of bacterial colonization of patients undergoing mechanical ventilation is firstly the oropharynx/upper gastrointestinal tract, followed by the lower respiratory tract, leading on to ETT colonization. Colonization of the ETT with microorganisms commonly causing nosocomial pneumonia appears to persist in many cases despite apparently successful treatment of the previous pneumonia.

This study confirms previous findings that the oropharynx is an important early site of colonization by bacterial pathogens in severely ill patients, which occurred in these cases, and has been demonstrated in many other studies by $48 \mathrm{~h}$ [7]. The next site at which micro-organisms were noted was in the upper gastrointestinal tract, occurring progressively between $36-60 \mathrm{~h}$. The present study suggests that colonization of the upper gastrointestinal tract is a common and early occurrence. The role of the stomach as a reservoir for nosocomial pneumonia is still a controversial topic. While some investigators suggest that gastric colonization is less important in the pathogenesis of ventilatorassociated pneumonia [11, 19], even in cases undergoing chronic mechanical ventilation [13], other meta-analyses [12] and reviews [20, 21] have concluded that there is major evidence in the literature in favour of the gastric origin of at least some pulmonary infections.

Thereafter colonizing micro-organisms were found in lower respiratory tract secretions obtained through sterile suctioning through the ETT. This was noted from as early as $36 \mathrm{~h}$, but was most prominent between $60-84 \mathrm{~h}$. Isolation of micro-organisms from within the ETT itself was noted from as early as $48 \mathrm{~h}$ (scanty isolation), while colonization in significant amounts was most apparent at $96 \mathrm{~h}$. It is interesting to note that the appearance of microorganisms in lower respiratory tract secretions of these patients almost invariably preceded their appearance in the interior of the ETT. This suggests that colonization of the ETT in these patients occurred from the lower respiratory tract, as has been suggested by others $[16,17]$. This 
conclusion is strengthened by two additional observations in this study. Firstly, ETT colonization occurred despite the use of heat and moisture exchangers (incorporating a bacterial filter) within the ventilator circuit of some of these cases, which would be expected to prevent direct contamination of the ETT from the exterior or from the ventilator tubing. These devices would not, however, be expected to prevent colonization occurring from the lower respiratory tract below. Secondly, colonization was invariably noted to occur in the distal part of the tube of all these patients, which is where such secretions would be expected to accumulate if they appeared in this site as a result of direct spread of secretions from the lower respiratory tract by means of the mucociliary escalator.

A definite pattern to the colonization process emerged with the various micro-organisms, even though there were small patient numbers. There appeared to be a tendency for Gram-positive isolates to colonize the oropharynx early, between $12-36 \mathrm{~h}$, and to appear rapidly thereafter in the stomach and then in lower respiratory tract secretions. No Gram-positive isolates were found to colonize the ETT in significant amounts. Colonization of the oropharynx with $A$. anitratus occurred later, between $36-48 \mathrm{~h}$ and these organisms progressively colonized the stomach, the lower respiratory tract and then the ETT (by $96 \mathrm{~h}$ ). The pattern of colonization with the other micro-organisms was less clearcut. Figure 2 shows the sequence of colonization of the various sites in four patients colonized with $A$. anitratus and four cases colonized with staphylococcal isolates.

On SEM, it was demonstrated that the micro-organisms colonizing the interior of the ETT adhere within a biofilm (fig. 1a and b). In 1986, SotTILE et al. [14] systematically studied 25 patients in ICU undergoing extubation and found that in $84 \%$ of cases the ETT was completely covered and in $16 \%$ partly covered by an amorphous bacteriumcontaining matrix. They were among the first to suggest that there may be a relationship between nosocomial pulmonary infections and bacterial adherence to the interior of the ETT. A study in neonates undergoing prolonged mechanical ventilation failed to demonstrate a clinical correlation between ETT colonization and nosocomial pneumonia [15]. Nevertheless, others have suggested that the biofilm may not only represent a mechanism of tube obstruction, but also be a source of nosocomial pulmonary infection [14-17]. It has been reported that organisms adhering in the biofilm in the interior of the ETT may enter the lungs via aerosolization of particles during the ventilatory process or following mechanical dislodging of these secretions at the time of airway suctioning [5, 6, 14-17].

Nosocomial pneumonia occurred in a total of 13 patients in these two study groups. Because of the study design, it was only possible to relate the time of ETT colonization to development of nosocomial pneumonia in patients enrolled in the second part of this study. Three of these patients developed nosocomial pneumonia. In one of these, no organisms were noted in the ETT. In a second case, significant colonization of the lower respiratory tract and ETT had occurred simultaneously with A. anitratus at $60 \mathrm{~h}$, followed by clinical evidence of nosocomial pneumonia, deemed to be due to the same organism, at $96 \mathrm{~h}$. In a third patient, colonization of the lower respiratory tract with $A$. anitratus occurred at $72 \mathrm{~h}$, followed by ETT colonization with the same organism at $84 \mathrm{~h}$, followed by nosocomial pneumonia, deemed to be due to the same organism, at 96 h. Thus, in two of these cases, nosocomial pneumonia followed ETT colonization. In the first part of the study, 10 patients developed pneumonia and in six of these, despite apparently successful treatment of the infection, at least one of the micro-organisms deemed to have been responsible for the pneumonia was still found colonizing the ETT on extubation. Thus, persistence of micro-organisms in the ETT occurred in several cases even after apparently successful treatment of the previous nosocomial pneumonia. While this series is far too small to make any definitive comment, it would be interesting to speculate that colonization of the ETT may precede nosocomial pneumonia in a number of patients or, more importantly, be a persistent source of organisms that may cause recurrent pneumonia, much in the same way as it has been suggested in recent studies that recurrent $P$. aeruginosa pneumonia may be due to persistence and relapse of these strains in the lower respiratory tract [22].

With regard to the management of ETT biofilm formation and colonization, a number of options have been considered. While regular ETT changes may seem appropriate, recent studies have suggested that this may be associated with a higher incidence of nosocomial pneumonia $[4,22]$. The reasons for this are not entirely clear, but may relate to the passing of the airway access tube through areas (e.g. the naso- or oropharynx) that are already colonized in critically ill cases with common nosocomial pathogens. Others have suggested that the interior of the ETT could be "brushed" using a specially developed wire mesh instrument or that specific materials could be developed for airway access tubes that impede biofilm formation, these being steps which may prevent nosocomial pneumonia [5, $16,17]$.

In conclusion, it has been noted that endotracheal tube colonization and biofilm formation occurs in many patients undergoing mechanical ventilation, from a very early stage. Biofilm formation may in many cases precede the development of nosocomial pneumonia, and perhaps more importantly, represent a persistent source of organisms causing recurrent infections. Further studies are needed to clarify the exact role of endotracheal tube colonization in the pathogenesis of nosocomial pneumonia, since measures aimed at addressing the problem of endotracheal tube colonization and biofilm formation may help impact further on this ongoing problem.

Acknowledgements. The authors would like to thank J-P. Tissandie, P. Mahida and J. Omar for their help with this study.

\section{References}

1. Johanson WG, Pierce AK, Sanford JP, et al. Nosocomial respiratory infections with Gram-negative bacilli: the significance of colonization of the respiratory tract. Ann Intern Med 1972; 77: 701-706.

2. Chastre J, Trouillet JL. Nosocomial pneumonia. Curr Opin Infect Dis 1995; 1: 194-201.

3. A'Court C, Garrard CS. 1-Nosocomial pneumonia in the intensive care unit: mechanisms and significance. Thorax 1992; 47: 465-473.

4. Craven DE, Steger KA. Nosocomial pneumonia in the intubated patient: new concepts on pathogenesis and prevention. Infect Dis Clinic North Am 1989; 3: 843-866. 
5. Campbell DG Jr, Niederman MS, Broughton WA, et al. Hospital-acquired pneumonia in adults: diagnosis, assessment of severity, initial antimicrobial therapy, and preventative strategies. Am J Respir Crit Care Med 1995; 153: 1711-1725.

6. Craven DE, Steger KA. Epidemiology of nosocomial pneumonia: new perspectives of an old disease. Chest 1995; 108 (Suppl.): 1S-16S.

7. Estes RJ, Meduri GU. The pathogenesis of ventilatorassociated pneumonia: I. Mechanisms of bacterial transcolonization and airway innoculation. Intensive Care Med 1995; 21: 365-383.

8. Niederman MS. Bacterial adherence as a mechanism of airway colonization. Eur J Clin Microbiol Infect Dis 1989; 8: 15-20.

9. Niederman MS. The pathogenesis of airway colonization: lessons learnt from the study of bacterial adherence. Eur Respir J 1994; 17: 1737-1740.

10. de Latorre FJ, Pont T, Ferrer A, et al. Pattern of tracheal colonization during mechanical ventilation. Am J Respir Crit Care Med 1995; 152: 1028-1033.

11. Bonten MJM, Gaillard CA, van Tiel FH, Smeets HEW, van der Geest S, Stobberingh EE. The stomach is not a source for colonization of the upper respiratory tract and pneumonia in ICU patients. Chest 1994; 105: 878-884.

12. Heyland D, Mandell LA. Gastric colonization by Gramnegative bacilli and nosocomial pneumonia in the intensive care unit: evidence for causation. Chest 1992; 101: 187-193.

13. Palmer LB, Donelan SV, Fox G, Bellemore E, Greene WH. Gastric flora in the chronically mechanically ventilated patients: relationship to upper and lower airway colonization. Am J Respir Crit Care Med 1995; 151: 1063-1067.
14. Sottile FD, Marrie TJ, Prough DS, et al. Nosocomial pulmonary infection: possible aetiologic significance of bacterial adhesion to the endotracheal tube. Crit Care Med 1986; 14: 265-270.

15. Diaz-Blanco J, Clawson RC, Roberson SM, et al. Electron microscopic evaluation of bacterial adherence to polyvinyl chloride endotracheal tubes used in neonates. Crit Care Med 1989; 17: 1335-1340.

16. Inglis TJJ, Millar MR, Jones JG, et al. Tracheal tube biofilm as a source of bacterial colonization of the lung. $J$ Clin Microbiol 1989; 27: 2014-2018.

17. Inglis TJJ, Tit-Meng L, Mah-Lee $\mathrm{N}$, et al. Structural features of tracheal tube biofilm, formed during prolonged mechanical ventilation. Chest 1995; 108: 10491052.

18. Goldstein JI, Yakowitz H, eds. Practical Scanning Electron Microscopy. Electron and Ion Microprobe Analysis. New York, Plenum Press, 1997.

19. Bonten MJM, Gaillard CA, de Leeuw PW, Stobberingh EE. Role of colonization of the upper intestinal tract in the pathogenesis of ventilator-associated pneumonia. Clin Infect Dis 1997; 24: 309-319.

20. Torres A, El-Ebiary M, Soler N, Monton C, Fabegras N, Hernandez C. Stomach as a source of colonization of the respiratory tract during mechanical ventilation: association with ventilator-associated pneumonia. Eur Respir $J$ 1996; 9: 1729-1735.

21. Torres A, Aznor R, Gatell JM, et al. Incidence, risk, and prognosis factors of nosocomial pneumonia in mechanically ventilated patients. Am Rev Respir Dis 1990; 142: 523-528.

22. Rello J, Mariscal D, March F, et al. Recurrent Pseudomonas aeruginosa pneumonia in ventilated patients. Am J Respir Crit Care Med 1998; 157: 912-916. 